\title{
Construction of lower and upper solutions for first-order periodic problem
}

Ruyun Ma* and Lu Zhang

"Correspondence: mary@nwnu.edu.cn Department of Mathematics, Northwest Normal University Lanzhou, 730070, P.R. China

\section{然 Springer}

\begin{abstract}
In this paper, we construct nonconstant lower and upper solutions for the periodic boundary value problem $x^{\prime}+f(t, x)=e(t), x(0)=x(T)$ and find their estimates. We prove the existence of positive solutions for the singular problem $x^{\prime}+g(x)=e(t)$, $x(0)=x(T)$ by using these results.
\end{abstract}

MSC: $34 \mathrm{~B} 10 ; 34 \mathrm{~B} 18$

Keywords: periodic boundary value problem; singular problem; lower and upper solutions

\section{Introduction}

The method of lower and upper solutions is an elementary but powerful tool in the existence theory of solutions to initial value problems and for periodic boundary value problems, even in cases where no special structure is assumed on the nonlinearity. Starting with the pioneering work of Moretto [1] for locally Lipschitzian ordinary differential equations, the method of upper and lower solutions for the periodic boundary value problem

$$
x^{\prime}+f(t, x)=0, \quad x(0)=x(T)
$$

has been extended to the case of a continuous right-hand side $f:[0, T] \times \mathbb{R} \rightarrow \mathbb{R}$ (see, e.g., [2-6]).

In Franco et al. [6], the method of lower and upper solutions was applied to the periodic problem

$$
x^{\prime}=f(t, x), \quad x(0)=x(T)
$$

and they also obtained a similar result to Lemma 1.1 below.

In order to apply these results, finding upper and lower solutions is very important. But the problem of construction of lower and upper solutions has been solved very rarely (see, e.g., $[7,8])$. In this paper we fill this gap and present conditions ensuring the existence of nonconstant lower and upper solutions to the first-order periodic boundary value problem (1.1) and find their estimates. This enables us to prove the existence result for the periodic problem with strong singularity.

(c) $2015 \mathrm{Ma}$ and Zhang. This article is distributed under the terms of the Creative Commons Attribution 4.0 International License (http://creativecommons.org/licenses/by/4.0/), which permits unrestricted use, distribution, and reproduction in any medium, provided you give appropriate credit to the original author(s) and the source, provide a link to the Creative Commons license, and indicate if changes were made. 
In order to explain the main results of the paper, let us introduce some notation: $C[0, T]$ stands for the set of functions continuous on $[0, T]$. For $x \in C[0, T]$, we denote

$$
\bar{x}=\frac{1}{T} \int_{0}^{T} x(s) d s, \quad\|x\|_{1}=\int_{0}^{T}|x(s)| d s .
$$

If $x \in C[0, T]$, then we write $x^{+}(t)=\max \{x(t), 0\}$ and $x^{-}(t)=\max \{-x(t), 0\}$. For $e \in$ $C[0, T]$, we put

$$
E=\int_{0}^{T} e(t) d t, \quad E_{ \pm}=\int_{0}^{T} e^{ \pm}(t) d t
$$

and note that $E=E_{+}-E_{-}$.

Let us recall first some classical definitions and results. They are taken from [3].

Definition 1.1 A lower solution $\alpha$ (respectively, an upper solution $\beta$ ) of problem (1.1) is a function $\alpha \in C^{1}[0, T]$ such that

$$
\alpha^{\prime}+f(t, \alpha) \geq 0, \quad t \in[0, T], \quad \alpha(0) \geq \alpha(T)
$$

(respectively, $\beta \in C^{1}[0, T]$ and

$$
\left.\beta^{\prime}(t)+f(t, \beta) \leq 0, \quad t \in[0, T], \quad \beta(0) \leq \beta(T)\right)
$$

The basic existence theorem of the method of upper and lower solutions for (1.1) can be stated as follows.

Lemma 1.1 If problem (1.1) has a lower solution $\alpha$ and upper solution $\beta$ such that $\alpha(t) \leq$ $\beta(t)$ for all $t \in[0, T]$ (resp. $\beta(t) \leq \alpha(t)$ for all $t \in[0, T]$ ), then problem (1.1) has at least one solution $x$ such that $\alpha(t) \leq x(t) \leq \beta(t)$ for all $t \in[0, T]($ resp. $\beta(t) \leq x(t) \leq \alpha(t)$ for all $t \in[0, T])$.

The paper is organized as follows. In Section 2 we develop a method to construct lower and upper solutions for (1.1). As the application, in Section 3, we establish an existence result for a nonlinear first-order periodic problem with strong singularity.

\section{Construction of lower and upper solutions}

In this section we consider

$$
x^{\prime}+f(t, x)=e(t), \quad x(0)=x(T)
$$

where $f:[0, T] \times \mathbb{R} \rightarrow \mathbb{R}$ and $e:[0, T] \rightarrow \mathbb{R}$ are continuous.

Let us consider an auxiliary boundary value problem

$$
x^{\prime}+\delta(t)=0, \quad x(0)=x(T)=\zeta
$$

where $\delta \in C[0, T]$ satisfies $\bar{\delta}=0$ and $\zeta \in \mathbb{R}$. 
Clearly, for all $\zeta \in \mathbb{R}$, problem (2.2) possesses a unique solution $x_{\zeta}$ as follows:

$$
x_{\zeta}(t)=\zeta-\int_{0}^{t} \delta(s) d s
$$

Proposition 2.1 Assume that there are $B_{1} \in \mathbb{R}, c_{1} \in C[0, T]$ such that

$$
f(t, x) \leq c_{1}(t) \quad \text { for all } t \in[0, T] \text { and } x \in\left[B_{1}, B_{1}+2\left\|\eta_{1}\right\|_{1}\right]
$$

where $\eta_{1}(t)=\phi^{-}(t)-\phi^{+}(t) \frac{\Phi_{-}}{\Phi_{+}}$and $\phi=c_{1}-e$. If

$$
\bar{c}_{1}-\bar{e} \leq 0,
$$

then there exists an upper solution $\beta$ of (2.1) such that

$$
B_{1} \leq \beta \leq B_{1}+2\left\|\eta_{1}\right\|_{1} \text { in }[0, T]
$$

Proof Consider the function $\phi=c_{1}-e$. We have two cases.

Case 1. Assume that $\Phi_{+}=\int_{0}^{T} \phi^{+}(t) d t=0$. Taking $\beta=\xi \in\left[B_{1}, B_{1}+2\left\|\eta_{1}\right\|_{1}\right]$ and using $c_{1}-e \leq 0$, it follows from (2.3) that $\beta$ is an upper solution of (2.1).

Case 2. Assume that $\Phi_{+}>0$. Using

$$
\int_{0}^{T}\left[\phi^{+}(t) \Phi_{-}-\phi^{-}(t) \Phi_{+}\right] d t=0
$$

it follows that there exists $\omega$ such that

$$
\omega^{\prime}(t)+\phi^{+}(t) \Phi_{-}-\phi^{-}(t) \Phi_{+}=0, \quad \omega(0)=\omega(T)=\zeta .
$$

Taking $x_{0}=\frac{1}{\Phi_{+}}$and

$$
\beta(t)=\zeta-\int_{0}^{t} x_{0}\left[\phi^{+}(s) \Phi_{-}-\phi^{-}(s) \Phi_{+}\right] d s
$$

it is easy to see that $\beta(0)=\beta(T)$. Since $\left|-\int_{0}^{t} x_{0}\left[\phi^{+}(s) \Phi_{-}-\phi^{-}(s) \Phi_{+}\right] d s\right|=\left|\int_{0}^{t} \eta_{1}(s) d s\right| \leq$ $\left\|\eta_{1}\right\|_{1}$, choosing $\zeta=B_{1}+\left\|\eta_{1}\right\|_{1}$, we obtain

$$
B_{1} \leq \beta(t) \leq B_{1}+2\left\|\eta_{1}\right\|_{1}
$$

which means that (2.5) holds.

Now, using (2.4), it follows that $\Phi_{+} \leq \Phi_{-}$, implying that

$$
\beta^{\prime}(t)=x_{0} \omega^{\prime}(t)=-x_{0}\left[\phi^{+}(s) \Phi_{-}-\phi^{-}(s) \Phi_{+}\right] \leq-\phi(t)=e(t)-c_{1}(t) .
$$

From (2.3) and (2.5) we deduce that

$$
\beta^{\prime}(t)+f(t, \beta) \leq \beta^{\prime}(t)+c_{1}(t) \leq e(t) \quad \text { for all } t \in[0, T],
$$

and the proof is completed. 
Using similar arguments, one can prove the following proposition.

Proposition 2.2 Assume that there are $B_{2} \in \mathbb{R}, c_{2} \in C[0, T]$ such that

$$
f(t, x) \geq c_{2}(t) \text { for all } t \in[0, T] \text { and } x \in\left[B_{2}, B_{2}+2\left\|\eta_{2}\right\|_{1}\right] \text {, }
$$

where $\eta_{2}(t)=\phi^{-}(t)-\phi^{+}(t) \frac{\Phi_{-}}{\Phi_{+}}$and $\phi=c_{2}-e$. If

$$
\bar{c}_{2}-\bar{e} \geq 0,
$$

then there exists a lower solution $\alpha$ of (2.1) such that

$$
B_{2} \leq \alpha \leq B_{2}+2\left\|\eta_{2}\right\|_{1} \quad \text { in }[0, T]
$$

Theorem 2.1 and Theorem 2.2 are simple examples of existence results which follow immediately from Lemma 1.1, Propositions 2.1 and 2.2.

Theorem 2.1 Assume that there are $B_{i} \in \mathbb{R}, c_{i} \in C[0, T]$ such that

$$
(-1)^{i+1} f(t, x) \leq(-1)^{i+1} c_{i}(t), \quad(-1)^{i+1}\left(\bar{c}_{i}-\bar{e}\right) \leq 0
$$

for all $t \in[0, T], x \in\left[B_{i}, B_{i}+2\left\|\eta_{i}\right\|_{1}\right]$ and all $i \in\{1,2\}$,

$$
B_{2}+2\left\|\eta_{2}\right\|_{1} \leq B_{1}
$$

Then problem (2.1) possesses a solution $x$ such that

$$
B_{2} \leq x(t) \leq B_{1}+2\left\|\eta_{1}\right\|_{1}
$$

$\left(\eta_{1}, \eta_{2}\right.$ are given by Proposition 2.1 and Proposition 2.2, respectively).

Theorem 2.2 Assume that there are $B_{1}, B_{2} \in \mathbb{R}, c_{1}, c_{2} \in C[0, T]$ such that the assumptions of Theorem 2.1 are satisfied with

$$
B_{1}+2\left\|\eta_{1}\right\|_{1} \leq B_{2}
$$

instead of (2.6). Then problem (2.1) possesses a solution $x$ such that

$$
B_{1} \leq x(t) \leq B_{2}+2\left\|\eta_{2}\right\|_{1}
$$

Now, we consider problem (1.1), i.e., $e \equiv 0$.

Proposition 2.3 Assume that there are $A_{1} \in \mathbb{R}, b_{1} \in C[0, T]$ such that $\bar{b}_{1}=0$ and

$$
f(t, x) \geq b_{1}(t) \quad \text { for all } t \in[0, T] \text { and } x \in\left[A_{1}, A_{1}+2\|b\|_{1}\right] .
$$

Then there exists a lower solution $\alpha$ of (1.1) such that

$$
A_{1} \leq \alpha \leq A_{1}+2\left\|b_{1}\right\|_{1} \quad \text { in }[0, T] .
$$


Proof Problem (2.2) with $\delta(t)=b_{1}(t)$ on $[0, T]$ has a unique solution

$$
x_{\zeta}(t)=\zeta-\int_{0}^{t} b_{1}(s) d s .
$$

Since $-\left\|b_{1}\right\|_{1} \leq-\int_{0}^{t} b_{1}(s) d s \leq\left\|b_{1}\right\|_{1}$, choosing $\zeta=A_{1}+\left\|b_{1}\right\|_{1}$, we obtain

$$
A_{1} \leq x_{\zeta}(t) \leq A_{1}+2\left\|b_{1}\right\|_{1}
$$

Let us take $\alpha(t)=x_{\zeta}(t)$, which means that (2.8) holds. According to (2.7) this yields

$$
0=\alpha^{\prime}(t)+b_{1}(t) \leq \alpha^{\prime}(t)+f(t, \alpha) \quad \text { on }[0, T]
$$

Furthermore, we have $\alpha(0)=\alpha(T)$, by Definition 1.1, the function $\alpha$ is a lower solution of (1.1).

The following assertion is due to Proposition 2.1 and its proof can be omitted.

Proposition 2.4 Assume that there are $A_{2} \in \mathbb{R}, b_{2} \in C[0, T]$ such that $\bar{b}_{2}=0$ and

$$
f(t, x) \leq b_{2}(t) \quad \text { for all } t \in[0, T] \text { and } x \in\left[A_{2}, A_{2}+2\left\|b_{2}\right\|_{1}\right]
$$

Then there exists an upper solution $\beta$ of (1.1) such that

$$
A_{2} \leq \beta \leq A_{2}+2\left\|b_{2}\right\|_{1} \quad \text { in }[0, T] .
$$

It is easy to see that the following results can be shown in a similar manner as in Theorem 2.1.

Theorem 2.3 Assume that there are $A_{i} \in \mathbb{R}, b_{i} \in C[0, T]$ such that $\bar{b}_{i}=0$ and

$$
(-1)^{i} f(t, x) \leq(-1)^{i} b_{i}(t)
$$

for all $t \in[0, T], x \in\left[A_{i}, A_{i}+2\left\|b_{i}\right\|_{1}\right]$ and all $i \in\{1,2\}$,

$$
A_{2}+2\left\|b_{2}\right\|_{1} \leq A_{1}
$$

Then problem (1.1) possesses a solution $x$ such that

$$
A_{2} \leq x(t) \leq A_{1}+2\left\|b_{1}\right\|_{1}
$$

Theorem 2.4 Assume that there are $A_{1}, A_{2} \in \mathbb{R}, b_{1}, b_{2} \in C[0, T]$ such that the assumptions of Theorem 2.3 are satisfied with

$$
A_{1}+2\left\|b_{1}\right\|_{1} \leq A_{2}
$$

instead of (2.10). Then problem (1.1) possesses a solution $x$ such that

$$
A_{1} \leq x(t) \leq A_{2}+2\left\|b_{2}\right\|_{1} .
$$


Problem (1.1) has been considered by several authors (see, e.g., [6, 9]). In [9], Peng gave the existence result as follows.

Theorem A Assume that there exists a positive number $M$ such that $M x-f(t, x) \geq 0$ for $t \in[0, T]$ and $x \geq 0$. If

(i) $f_{0}=\liminf _{x \rightarrow 0^{+}} \min _{t \in[0, T]} \frac{f(t, x)}{x}>0$ and $f^{\infty}=\limsup _{x \rightarrow \infty} \max _{t \in[0, T]} \frac{f(t, x)}{x}<0$; or

(ii) $f_{\infty}=\liminf _{x \rightarrow \infty} \min _{t \in[0, T]} \frac{f(t, x)}{x}>0$ and $f^{0}=\lim \sup _{x \rightarrow \infty} \max _{t \in[0, T]} \frac{f(t, x)}{x}<0$.

Then problem (1.1) has at least one positive solution.

In this paper, we do not require $f$ to satisfy (i) or (ii). Now we give an example.

Example 2.1 Consider the periodic boundary value problem

$$
x^{\prime}+\arctan (x-1)=0, \quad x(0)=x(1) .
$$

It is easy to see that

$$
f_{0}=\lim _{x \rightarrow 0^{+}} \frac{f(x)}{x}=-\infty \text { and } f_{\infty}=\lim _{x \rightarrow \infty} \frac{f(x)}{x}=0 .
$$

So we can not obtain existence result by Theorem A. But if we choose $b_{1}(t)=\frac{\pi}{3} t-\frac{\pi}{6}$, compute that $\bar{b}_{1}=\int_{0}^{1} \frac{\pi}{3} s-\frac{\pi}{6} d s=0$, and (2.7) holds for $A_{1}=\frac{\sqrt{3}}{3}+1$ and

$$
A_{1}+2\left\|b_{1}\right\|_{1}=\frac{\sqrt{3}}{3}+1+2 \int_{0}^{1}\left|\frac{\pi}{3} s-\frac{\pi}{6}\right| d s=\frac{\sqrt{3}}{3}+1+\frac{\pi}{6} .
$$

By Proposition 2.3, problem (2.11) has a lower solution. In fact,

$$
\alpha(t)=A_{1}+\left\|b_{1}\right\|_{1}-\int_{0}^{t} b_{1}(s) d s=-\frac{\pi}{6} t^{2}+\frac{\pi}{6} t+\frac{\pi}{12}+\frac{\sqrt{3}}{3}+1
$$

is a lower solution.

According to Proposition 2.4, we choose $b_{2}(t)=\frac{\pi}{4} t-\frac{\pi}{8}, A_{2}=1-\frac{\sqrt{3}}{3}-\frac{\pi}{8}$, compute that $A_{2}+2\left\|b_{2}\right\|_{1}=1-\frac{\sqrt{3}}{3}$ and (2.9) hold. Then problem (2.11) has an upper solution

$$
\beta(t)=A_{2}+\left\|b_{2}\right\|_{1}-\int_{0}^{t} b_{2}(s) d s=1-\frac{\sqrt{3}}{3}-\frac{\pi}{16}-\frac{\pi}{8}\left(t^{2}-t\right) .
$$

Obviously, $A_{2}+2\left\|b_{2}\right\|_{1}<A_{1}$. Thus, from Theorem 2.2, problem (2.11) has a solution $x(t)$ satisfying

$$
0<1-\frac{\sqrt{3}}{3}-\frac{\pi}{8} \leq \beta(t) \leq x(t) \leq \alpha(t) \leq \frac{\sqrt{3}}{3}+1+\frac{\pi}{6}, \quad \forall t \in[0,1],
$$

i.e., $x$ is a positive solution of (2.11).

\section{Periodic problem with strong singularity}

We will consider the following singular equation with periodic conditions:

$$
x^{\prime}(t)+g(x)=e(t), \quad x(0)=x(T),
$$


where $g \in C(0, \infty), e \in C[0, T]$ and $g$ has strong singularity at 0 , i.e.,

$$
\lim _{x \rightarrow 0^{+}} \int_{x}^{1} g(\xi) d \xi=\infty
$$

Theorem 3.1 Assume that (3.2) is fulfilled, there exists $\tilde{A}_{1} \in(1, \infty)$ such that

$$
g(x) \leq \bar{e} \quad \text { for all } x \in\left[\tilde{A}_{1}, \tilde{B}_{1}\right]
$$

where

$$
\tilde{B}_{1}-\tilde{A}_{1}=2\|\eta\|_{1}, \quad \text { and } \quad \eta(t)=\phi^{-}(t)-\phi^{+}(t) \frac{\Phi_{-}}{\Phi_{+}}, \quad \phi=\bar{e}-e .
$$

Then problem (3.1) has a positive solution.

Proof If $g \in C(0, \infty)$ satisfies (3.2), then $\lim \sup _{x \rightarrow 0_{+}} g(x)=\infty$, which implies the existence of a sequence $\left\{\varepsilon_{n}\right\}_{n=1}^{\infty} \subset(0,1)$ such that

$$
g\left(\varepsilon_{n}\right)>0 \quad \text { for all } n \in \mathbb{N}, \quad \lim _{n \rightarrow \infty} \varepsilon_{n}=0, \quad \lim _{n \rightarrow \infty} g\left(\varepsilon_{n}\right)=\infty .
$$

Let $e_{M}=\max _{t \in[0, T]} e(t)$. By (3.3), there exists $N$ such that

$$
g\left(\varepsilon_{n}\right)>e_{M} \text { for all } n>N \text {. }
$$

We choose $\varepsilon=\varepsilon_{N+1}$ such that $g(\varepsilon)>e_{M}$. Let $R \geq \tilde{B}_{1}$ be a constant. For the proof of Theorem 3.1, we deal with the auxiliary family of problems

$$
x^{\prime}+\tilde{g}(x)=e(t), \quad x(0)=x(T),
$$

where

$$
\tilde{g}(x)= \begin{cases}0 & \text { if } x<0, \\ g(\varepsilon) \frac{x}{\varepsilon} & \text { if } x \in[0, \varepsilon], \\ g(x) & \text { if } x \in[\varepsilon, R] \\ g(R) & \text { if } x \geq R .\end{cases}
$$

Then $\tilde{g}(x)$ fulfils the assumptions of Proposition 2.1 with $c_{1}=\bar{e}$. Thus, by Proposition 2.1, problem (3.4) has an upper solution $\beta$ such that

$$
\tilde{A}_{1} \leq \beta \leq \tilde{B}_{1}
$$

Since $\varepsilon<\tilde{A}_{1} \leq \tilde{B}_{1} \leq R$, then $\varepsilon<\beta \leq R$ is a lower solution of (3.1).

It is easy to see that $\alpha=\varepsilon$ is a lower solution of (3.1). Thus, by Lemma 1.1, problem (3.1) has a positive solution $x$ such that $0<\varepsilon<x \leq B_{1}$.

Example 3.1 Consider the periodic boundary value problem with strong singularity

$$
x^{\prime}+\frac{1}{x}=e(t), \quad x(0)=x(T) .
$$


Let $g(x)=\frac{1}{x}, e(t)=t$. We can prove that $g(x)$ satisfies condition (3.2). Since $\bar{e}=\int_{0}^{1} t d t=\frac{1}{2}$, then $\widehat{\phi}=\bar{e}-e=\frac{1}{2}-t$.

We have

$$
\eta(t)=\widehat{\phi}^{-}(t)-\widehat{\phi}^{+}(t) \frac{\widehat{\Phi}_{-}}{\widehat{\Phi}_{+}}=-\widehat{\phi}=t-\frac{1}{2}, \quad\|\eta\|_{1}=\frac{1}{4} .
$$

We can choose $A_{1}=2$, then $B_{1}=A_{1}+2\|\eta\|_{1}=\frac{5}{2}$ and $g$ satisfies

$$
g(x)=\frac{1}{x} \leq \frac{1}{2} \quad \forall x \in\left[2, \frac{5}{2}\right] .
$$

By Theorem 3.1, problem (3.6) has a positive solution.

In fact, $\alpha=\frac{1}{2}$ is a lower solution of (3.6).

$$
\beta(t)=\frac{11}{4}+\frac{1}{2} t^{2}-\frac{1}{2} t
$$

is an upper solution of (3.6).

\section{Competing interests}

The authors declare that there is no conflict of interests regarding the publication of this paper.

\section{Authors' contributions}

RM completed the main study, carried out the results of this article. LZ drafted the manuscript, checked the proofs and verified the calculation. All the authors read and approved the final manuscript.

\section{Acknowledgements}

The authors are very grateful to the anonymous referees for their valuable suggestions. This work was supported by NSFC (No. 11361054 and No. 11201378), SRFDP (No. 2012 6203110004), and Gansu provincial National Science Foundation of China (No. 1208RJZA258).

\section{Received: 18 November 2014 Accepted: 9 October 2015 Published online: 20 October 2015}

\section{References}

1. Moretto, S: Sull'esistenza di soluzioni periodiche per l'equazione $y^{\prime}=f(t, y)$. Ann. Univ. Ferrara 8, 61-67 (1958)

2. Bereanu, C, Mawhin, J: Upper and lower solutions for periodic problems: first order difference vs first order differential equations. In: Mathematical Analysis and Applications. AIP Conf. Proc., vol. 835, pp. 30-36. Am. Inst. Phys., Melville (2006) (Reviewer: Johnny Henderson) 34C25 (39A11)

3. Mawhin, J: First order ordinary differential equations with several periodic solutions. Z. Angew. Math. Phys. 38, 257-265 (1987)

4. Obersnel, F, Omari, P: Old and new results for first order periodic ODEs without uniqueness: a comprehensive analysis via lower and upper solutions. Adv. Nonlinear Stud. 4, 323-376 (2004)

5. Lakshmikantham, V, Leela, S: Existence and monotone method for periodic solutions of first order differential equations. J. Math. Anal. Appl. 91, 237-243 (1983)

6. Franco, D, Nieto, J, O'Regan, D: Upper and lower solutions for first order problems with nonlinear boundary conditions. Extr. Math. 18, 153-160 (2003)

7. Bereanu, C, Gheorghe, D, Zamora, M: Periodic solutions for singular perturbations of the singular $\phi$-Laplacian operator. Commun. Contemp. Math. 15, 1-22 (2013)

8. Rachünková, I, Tvrdý, M: Construction of lower and upper functions and their application to regular and singular periodic boundary value problems. Nonlinear Anal. 47, 3937-3948 (2001)

9. Peng, S: Positive solutions for first order periodic boundary value problem. Appl. Math. Comput. 158, 345-351 (2004) 